tigt werden, dem Akteur ebenfalls zur Verfügung stehen.

Den Öko-Bilanz-Forschern und Forscherinnen ist diese Problematik allgemein bewußt. Die Frage ist nur, ob dies auch für ihre Kunden aus der Industrie und der Politik gilt. Die Öko-Bilanz ist bei ihrem derzeitigen Forschungsstand ein komplexes Instrument für Fachexperten, das in seiner Anwendung eine Interpretation durch diese erfahren muß. Dabei fließt zwangsläufig die »Expertenmeinung « in die Expertise ein. So kann eine Öko-Bilanz von unterschiedlichen Experten auch unterśchiedlich interpretiert werden. Die Fachvertreter des Umweltbundesamtes können ein Lied davon singen, wie aus der gleichen Datenbasis unterschiedliche »Ergebnisse « herausgeholt werden können.

Dieses Problem wird sich auch nicht ändern, wenn einmal die standardisierte DIN-Produktbilanz kommt. Zwar sollen hier lobenswerterweise Aspekte wie Vollständigkeit, funktionale Vergleichbarkeit und Kommunikation aller Grundannahmen etc. als Mindestanforderungen festgelegt werden, doch lösen auch diese Anforderungen die oben genannten Probleme nur in Teilbereichen. Hinzu kommt, daß durch diese Anforderungen die Kommunikation solcher Bilanzen eher erschwert wird. Insbesondere für Menschen, die sich nicht alltäglich mit dieser Thematik auseinandersetzen, wird eine eigene Interpretation der Ergebnisse fast unmöglich. In diesem Sinne müssen Formen gefunden werden, die eine vernünftige Kommunikation der Ergebnisse auch gegenüber Laien ermöglichen. Hierbei geht es nicht nur um eine informationsvermittelnde Aufbereitung der Inhalte. Insbesondere in der Politikberatung und dem Verbraucherschutz geht es vielmehr auch um die Entwicklung von Prozeduren und/oder Gremien, die eine sinnvolle Nutzung ermöglichen.

\section{Hendric Hallay, Reinhard Pfriem Oldenburg}

\title{
Die Produktbilanz als ökologisches Informationsinstrument
}

Die deutsche Diskussion um Produktbilanzen wurde u. a. durch einen Methodenvorschlag des Umweltbundesamtes (1) beeinflußt, der die Schritte Zieldefinition, Sachbilanz, Wirkungsbilanz und Bewertung vorgibt. Die Weiterentwicklungen im Umweltbundesamt und den nationalen und internationalen Normungsausschüssen werden ausführlich im Artikel von $\mathrm{H}$. Neitzel ab Seite 9 beschrieben. Zur praktischen Erprobung dieser Methodik wurde eine mit großer Spannung erwartete Verpackungsmittelstudie in Auftrag gegeben. Unter anderem auf Grund methodischer Probleme blieb die Studie auf die Erstellung der Sachbilanz, d. h. die Datenerhebung, beschränkt. Die Frage der ökologischen Auswirkungen und deren Bewertung konnte bisher nicht abschließend geklärt werden. Doch selbst die Daten der Sachbilanz sind der Öffentlichkeit nicht zugänglich sondern harren im Umweltbundesamt der abschließenden Bewertung.

In Zusammenarbeit mit der ecco $\mathrm{GmbH}$ wurde am IÖW ein Instrument zur ökologischen Produktbilanzierung entwickelt, das auf der einen Seite die aktuellen Gegebenheiten und Probleme der Praxis reflektiert und auf der anderen Seite den theoretischen Ansprüchen, die u.a. im Methodenvorschlag des UBA formuliert wurden, genügt. Seit Ende 1992 wurde das Instrument in mehreren Projekten eingesetzt und weiterentwickelt. Anhand von Beispielen aus den folgenden Projekten soll die Methodik vorgestellt werden:

- ökologischer Systemvergleich von Ein- und Mehrweggeschirr im Messeeinsatz,

- Ökobilanz für einen Baustoff,

- ökologischer Vergleich von Verpflegungssystemen.

Im Aufbau lehnt sich die IÖW-Produktbilanz an den UBA-Vorschlag an. Entsprechend sind die folgenden Schritte enthalten:

- Definition der Ziele, des Untersuchungsgegenstandes und der Systemgrenzen,

- Sachbilanz,

\section{Wirkungsbilanz,}

Bewertung,

- Aufzeigen von Optimierungspotentialen.

Dieser letzte Schritt ist im UBA-Vorschlag nicht enthalten. U.E. ist dies jedoch ein wesentlicher Bestandteil, um die Ergebnisse von Produktbilanzen interpretieren zu können.

\section{Definition der Ziele, des Untersuchungs- gegenstandes und der Systemgrenzen}

Gerade im Hinblick auf die geforderte Transparenz und Nachvollziehbarkeit kommt diesem Schritt große Bedeutung zu. In der Zieldefinition wird das Erkenntnisinteresse offengelegt, d.h. will man bspw. mehrere Einweg- und Mehrweggeschirrteile hinsichtlich ihrer Umweltwirkungen vergleichen oder ein spezifisches Produkt wie einen Baustoff bzgl. potentieller Schwachstellen im Lebensweg beleuchten. Wichtig ist, an dieser Stelle die Rahmenbedingungen zu nennen, unter denen die Untersuchungsgegenstände betrachtet werden. Die Untersuchung von Ein- und Mehrweggeschirrteilen ergibt verschiedene Ergebnisse, wird der Einsatz auf Messen oder im Flugbetrieb zugrunde gelegt. Das Beispiel der Einund Mehrweggeschirrteile verdeutlicht einen weiteren wesentlichen Bestandteil dieses ersten Schrittes: die funktionale Gleichwertigkeit der zu untersuchenden Varianten muß hergestellt werden.

D.h., wieviele Einwegbecher entsprechen einer Porzellantasse, was ist also real vergleichbar. Unter Berücksichtigung der Umlaufzahlen, der Bruch- und Schwundquote, der Spüleinrichtungen etc, erhält man die Anzahl an Einwegbechern und Porzellantassen, die dieselbe Funktion erfüllen, die Darreichung von 1.000 heißen Getränken. Hier können Kriterien wie Produktlebensdauer, Reparaturfähigkeit usw. mit einbezogen werden, die ebenfalls zur Funktionalität eines Produktes gehören. Am genannten Beispiel wird deutlich, daß die Festlegung und Kommunikation der Systemgrenzen unerläßlich ist. Wird die Reinigung der Porzellantassen einbezogen, werden Spülmaschinen oder Handspülen zugrunde gelegt etc. ? Festlegungen dieser Art beeinflussen das Ergebnis und werden daher explizit dargestellt und begründet. Nicht zuletzt enthält dieser erste Schritt die Offenlegung und Beschreibung der Datenverarbeitung und -aggregation in der Wirkungsbilanz und Bewertung.

\section{Die Sachbilanz}

In der Sachbilanz werden die ökologisch relevanten Daten erfaßt und zusammengestellt. Mit spezifischen Fragebögen werden die Informationen erhoben und anschließend in Form von Ökokontenrahmen zusammengefaßt. Diese Systematik gewährleistet zum einen, daß alle relevanten Produktlebensstufen integriert werden, d. h. von der Rohstoffgewinnung über die Vorproduktion, die Produktion, den Gebrauch, die Transporte bis zur Entsorgung des Produktes. Auf der anderen Seite wird sichergestellt, daß alle wesentlichen Inputs und Outputs auf den einzelnen Lebensstufen untersucht werden. Auf der Inputseite sind das die einfließenden Roh-, Hilfs- und Betriebsstoffe sowie die benötigte Energie nach Energieträgern differenziert. Auf der Outputseite stehen die Produkte, Kuppelprodukte, Emissionen in die Medien Luft und Wasser und die festen Abfälle, die entsorgt werden müssen. Ein Problem, das in der Sachbilanz immer wieder auftaucht, ist das fehlender Daten.

Selbst bei einer Produktbilanz, die für ein oder mehrere Produkte eines Unternehmens erstellt wird, sind Bestandteile mitzubilanzieren, die vom Unternehmen nur bezogen werden und für die entsprechend keine Produktionsdaten vorliegen. Hier muß auf allgemeine Grundlagenstudien zurückgegriffen werden, wie die des Schweizer Bundesamtes für Umwelt, Wald und Landschaft (BUWAL). Diese Studie enthält eine umfassende Sachbilanz von Verpackungsmitteln (Aluminium, Glas, Weißblech, Wellpappe, Papier, verschiedene Kunststoffe), gibt jedoch den Produktionsstand Mitte der 80er Jahre wieder. Dadurch werden entweder ausschließlich veraltete Daten verwendet oder mit aktuellen 
unternehmensspezifischen verglichen. Vor diesem Hintergrund wird es umso bedauerlicher, daß die aktuelle Verpackungsmittelstudie des UBA wegen Verpflichtungen zur Geheimhaltung noch unveröffentlicht ist.

Bereits durch diese systematische Darlegung der Stoff- und Energieströme werden einige Schwachstellen und Ansatzpunkte für Optimierungen deutlich. Bei einem Produktvergleich weist jedoch häufig das eine Produkt Schwachstellen in einem Bereich, das andere Produkt in einem anderen Bereich auf, so daß eine differenzierte Auswertung der Sachbilanz mit Hilfe der anschließend erläuterten Wirkungsbilanz und Bewertung erforderlich wird.

\section{Wirkungsbilanz}

Bis zu dieser Stelle besteht innerhalb der Fachwelt weitgehend Konsens. In der Wirkungsbilanzdiskussion treffen jedoch verschiedene Ansichten aufeinander, u. a. die der Praktiker und der Theoretiker. Eine theoretisch anspruchsvolle, aber auf Grund der fehlenden Sachbilanzdaten, Wirkungszusammenhänge, politisch vorgegebenen, 'allgemein anerkannten Zielhierarchien usw. undurchführbare Methodik nützt Anwendern nichts. Beim IÖW/ecco-Ansatz steht wiederum die Handlungsorientierung im Mittelpunkt.

In der Wirkungsbilanz werden die Emissionen in die Medien Luft und Wasser sowie die festen Abfälle hinsichtlich ihrer Schadwirkungspotentiale zusammengefaßt. Schadstoffe, die wie $\mathrm{SO}_{2}$ und $\mathrm{NO}_{\mathrm{x}}$ mehrere Wirkungen haben, werden mehrfach genannt. Bei der Abschätzung der Schadwirkungspotentiale werden UrsachenWirkungs-Modelle der ökologischen Grundlagenforschung herangezogen. Zur Frage, welche Gewichtungsfaktoren in diesem Zusammenhang sinnvoll sind, sei hier auf den Artikel von Guinée, Heijungs (Seite 7) verwiesen. Die Um- weltwirkungen, die im Rahmen der IÖW/eccoMethode derzeit betrachtet werden, sind im Medium Luft:

- Luftbelastungen mit humantoxischer Wirkung;

- Treibhauseffekt;

- Ozonabbau;

- Versauerung.

Im Medium Wasser:

- Toxizität;

- Eutrophierung;

- Versalzung.

Als Raumknappheitskriterium:

- Deponievolumen, das belegt wird im Zuge der Entsorgung.

Es handelt sich um Wirkungspotentiale und nicht um tatsächlich gemessene Wirkungen, denn Produktbilanzen beziehen derzeit regionale oder lokale Vorbelastungen und Gegebenheiten nicht ein, sondern sind standortneutral.

\section{Bewertung}

Ob der folgende Schritt der Gewichtung von Schadstoffen innerhalb einer Wirkungskategorie anhand ihres Anteils an der jeweiligen potentiellen Umweltwirkung bereits eine Bewertung oder das Ergebnis der ökologischen Forschung ist und damit noch zur Wirkungsbilanz gehört, ist umstritten. Im IÖW/ecco-Ansatz wird diese Gewichtung als erster, quantitativer Aspekt der Bewertung angesehen. Je nach Modell, das man zugrunde legt, verschieben sich die Wirkungszusammenhänge zwischen einzelnen Schadstoffen. Darüber hinaus sind diese Modelle monokausale Vereinfachungen, die weder Synergieeffekte noch komplexere Zusammenhänge wiedergeben. Daher scheint es gerechtfertigt, an dieser Stelle von Bewertung zu sprechen.

Die einer Umweltwirkung zuzurechnenden Emissionen werden mit diesen Gewichtungsfaktoren mulipliziert und im Anschluß addiert zu einer Zahl je Umweltwirkung. Diese Wirkungsindikatoren können nun zum Vergleich von Produkten herangezogen werden. Es ist darauf zu achten, daß nur innerhalb einer Kategorie verglichen werden kann und nicht kategorieübergreifend, da jeweils unterschiedliche $\mathrm{Ge}$ wichtungsfaktoren zugrunde liegen. D.h. die Treibhauszahl des Verpflegungssystems Fernküche kann mit der Treibhauszahl des Systems Kantine verglichen werden, jedoch nicht mit der Versauerungszahl der Fernküche oder Kantine. Die maximale Aggregation ist durch die Anzahl der betrachteten Umweltwirkungen vorgegeben, man erhält demnach nicht eine Gesamt-Wirkungszahl sondern in unserem Fall acht. Dadurch wird das Ergebnis zwar etwas komplexer, auf der anderen Seite jedoch aussagekräftiger und differenzierter und dadurch dem Anspruch auf Nachvollziehbarkeit eher gerecht.

Die graphische Aufbereitung der Ergebnisse in Form von Block-, Tortendiagrammen u. ä. führt zu anschaulichen, verständlichen Aussagen. Die Abbildungen zeigen, wie diese Auswertungen aussehen können. Ihnen liegen keine konkreten Projektergebnisse zugrunde, sondern willkürlich gewählte Zahlen.

Neben diesen quantitativen Aspekten der Bewertung existieren Kriterien, die nicht in Zahlen ausgedrückt werden können, in einer ökologischen Beurteilung jedoch nicht fehlen sollten. Dazu zählen Faktoren wie Landschaftsverbrauch, Artenschwund, Anteil nachwachsender und nicht-nachwachsender Rohstoffe, Ressourcenintensität usw. Diese Liste ist je nach untersuchtem Produkt spezifisch zu ergänzen. Hier könnte auch eine Schnittstelle zu sozialen und ökonomischen Kriterien entstehen, die nicht zu einer Produktbilanz im engeren Sinne gehören. Will man Produktbilanzen jedoch bspw. in der Verbraucherinformation einsetzen, werden diese Kriterien eine ebenso große Rolle spielen.

Abbildung 2

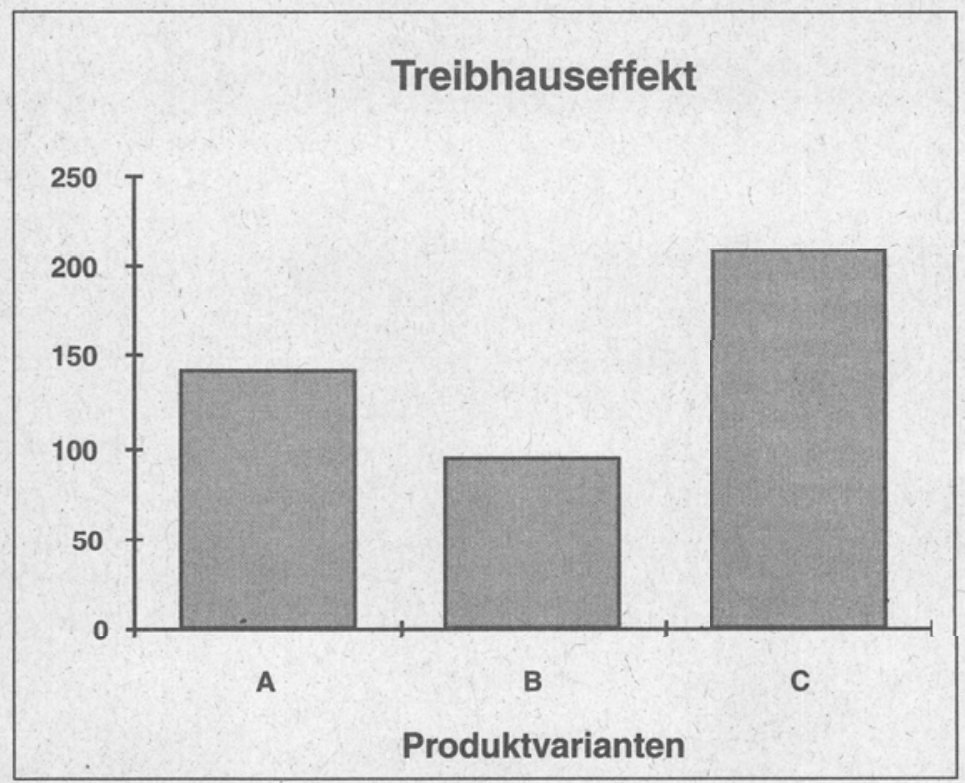

\section{Energieverbrauch in den Produktlebensphasen [MJ]}

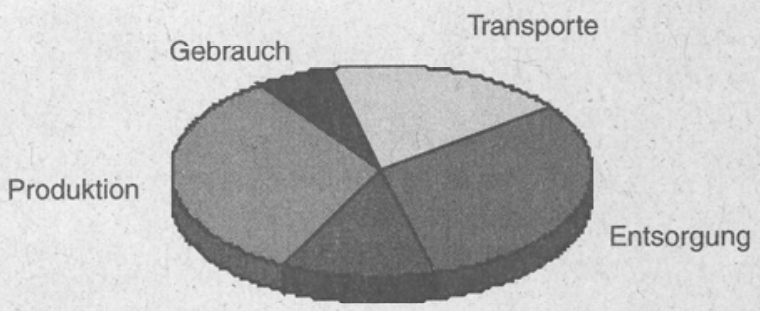

Rohstoffgewinnung 


\section{Aufzeigen von Optimierungspotentialen}

Wie bereits erwähnt wurde, ist ein wesentlicher Bestandteil der Produktbilanz nach IÖW/ecco die Handlungsorientierung. Deshalb werden über die Bewertung der Umweltwirkungen nach den oben genannten quantitativen und qualitativen Kriterien hinaus Optimierungspotentiale aufgezeigt. Das können Empfehlungen zur Gestaltung der Rahmenbedingungen sein. Will man bspw. auf Messen Mehrweggeschirr einsetzen, ist darauf $\mathrm{zu}$ achten, daß das Geschirr gründlich vorgesäubert wird, bevor es in die Spülmaschine kommt, um die Wasserbelastun- gen zu vermindern. Weiterhin sollten zur Verringerung des Energieverbrauchs bei der Wassererwärmung die Messestände mit Warmwasseranschlüssen für die Spülmaschinen versehen werden, um nur einige Beispiele zu nennen. Über Empfehlungen hinaus können Szenarien erstellt und im Hinblick auf ihre Umweltwirkungen durchgerechnet werden. Damit werden komplexere Sachverhalte und Zusammenhänge aufgedeckt und nachvollziehbạr gestaltet.

Bisher noch ungelöst ist die Frage, wie über Produktbilanzergebnisse kommuniziert werden soll und kann. Auf der einen Seite ist die Veröffentlichung durch Geheimhaltungsklauseln ein- geschränkt, auf der anderen Seite ist es bisher nicht gelungen, eine einheitliche Form zu finden, die Ergebnisse für verschiedene Interessengruppen kurz, verständlich und nachvollziehbar aufzubereiten. Diese Fragestellungen sollen in verschiedenen Forschungsprojekten des IÖW aufgegriffen und gelöst werden.

Kathrin Ankele, Berlin

(1) Biet, J. et al. (1992): Őkobilanzen für Produkte. Bedeutung - Sachstand - Perspektiven. Umweltbundesamt 38/92, Berlin

\section{Die Anwendung von Produktbilanzen}

Die Geschichte der Entwicklung und Anwendung von Produktbilanzen reicht bis in die 70er Jahre zurück. Allerdings wurden damals noch nicht die heute im deutschen Sprachraum am häufigsten verwendeten Begriffe »ProduktÖkobilanz« und »Produktlinienanalyse « - die beide erst Ende der 80er Jahre geprägt wurden und der international gebräuchlichste Begriff »Life-Cycle-Assessment « (LCA), der auch erst in den letzten Jahren den Durchbruch geschafft hat, benutzt. Die in der »Frühzeit« der Produktbilanzierung verwendeten Begriffe sind von allen typischen Merkmalen eines aufstrebenden Arbeitsgebietes gekennzeichnet:

- Verschiedene Autoren »kreieren« eigene Namen für ein Konzept, unabhängig davon, ob dieses Konzept schon einen Namen hat.

- Ein Konzept gleichen Namens wird von den verschiedenen Autoren unterschiedlich gehandhabt und ausgefüllt.

Mit Fug und Recht kann man die Situation der Frühzeit der Produktbilanzierung als eine Zeit einer gewissen »babylonischen Sprachverwirrung « und eines großen Methodenpluralismus kennzeichnen. Diese Zeit scheint heute überwunden zu sein.

Eine empirische Auswertung der ProduktbilanzStudien der letzten Jahre kann sich jedoch nicht ausschließlich auf den seit kurzem entwickelten Standard beschränken, denn damit würden eine große Anzahl von Arbeiten unberücksichtigt bleiben. Vielmehr muß auch die »Frühzeit « der Produktbilanzierung beachtet werden. Dies bedeutet, daß mitunter Arbeiten erfaßt werden, die aus heutiger Sicht wahrscheinlich anders beurteilt werden, zum Zeitpunkt ihrer Erstellung jedoch große Beachtung gefunden haben. Die empirische Basis beschränkt śich auf die uns aus der einschlägigen Diskussion bekannt gewordenen Studien in der eben erläuterten weiten begrifflichen Abgrenzung. Eine Anzahl von Arbeiten dürften dabei nicht berücksichtigt worden sein:

- Zum einen Arbeiten, die von Unternehmensberatungsbüros für Privatunternehmen er- stellt werden und von keiner der daran beteiligten Seiten publiziert werden. Beispielsweise hat die amerikanische Firma Franklin Associates bis Ende 1991 rund 70 Studien erstellt, von denen aber nur vier bekannt geworden sind.

- Zum anderen Arbeiten, die im Aussland erstellt worden sind und aufgrund der Sprache nicht in die internationale (englischsprachige) Diskussion eingegangen sind.

Gleichwohl ist die empirische Basis von 200 Arbeiten ausreichend, um diese nach einigen Merkmalen auszuwerten. Die Arbeiten, auf die wir uns beziehen, sind im einzelnen in einer $\mathrm{Bi}$ bliographie aufgelistet, die wir erstellt haben und zweimal jährlich aktualisieren. Diese Bibliographie ist als IÖW-Schriftenreihe 70/94 in einer Neuauflage erschienen.

Produktbilanz-Studien werden seit 1974 erstellt; sie nahmen eine langsame Entwicklung bis Ende der 80er Jahre. Erst Ende der 80er Jahre hat die »Hochzeit « eingesetzt. 1992 und 1993 hat sich ein gewisser Abschwung gezeigt, der möglicherweise auf die konjunkturelle Entwicklung zurückzuführen ist. Als eine Trendwende sollte dieser Rückgang an Studien jedoch nicht interpretiert werden. Produktbilanzen werden hauptsächlich bisher in der Bundesrepublik und in der Schweiz erstellt. Danach folgen die USA. Relativ wenig Bedeutung haben sie bisher in den südeuropäischen Mitgliedsstaaten der Europäischen Union, wenngleich in Italien wohl auch bedingt durch die Absicht, den $»$ Anschluß« nach Mitteleuropa zu schaffen - das Thema an Bedeutung gewinnt.

Die Themen bzw. Untersuchungsobjekte von Produktbilanzen konzentrieren sich auf einige Bereiche: Dominant dabei ist, wie die Tabelle zeigt, der Bereich der Verpackungen, auf den immerhin rund $40 \%$ aller Studien entfallen. Der Grund für die Dominanz dieses Bereiches liegt in der Kritik an den Verpackungen selbst, wie er speziell von Umweltorganisationen geübt worden ist. Unternehmen reagierten darauf mit der Erstellung von Studien, die sowohl der Recht- fertigung wie auch der echten Verbesserung der Verpackungen dienten.

Neben den Verpackungen sind die Bereiche der Hygiene- und Reinigungsprodukte (darunter speziell auch Windeln), der Kunststoffgranulate sowie der Baumaterialien bedeutsam. Dieser Darstellung ist jedoch nicht die Entwicklung der Themen zu entnehmen. Eine Analyse der Entwicklung der letzten Jahre zeigt, daß die Bedeutung des Verpackungsbereiches abgenommen hat: Wurden 1988/89 noch $53 \%$ aller Arbeiten zu diesem Thema gemacht, so reduzierte sich die Anzahl auf $27 \%$ in den Jahren 1992/93. Produktbilanzen werden in anderen Bereichen angewendet: Insbesondere der Bereich der Untersuchung von Kunststoff(granulaten) als Halbfertigprodukte hat zugenommen (Studien von Boustead u. a.). Weitere Bereiche, die leicht an Bedeutung zugenommen haben, sind elektrotechnische Produkte, Energie, Geschirr sowie Verkehr.

Daran ist abzulesen, daß Produktbilanz-Studien in neue Bereiche vorgedrungen sind, also eine

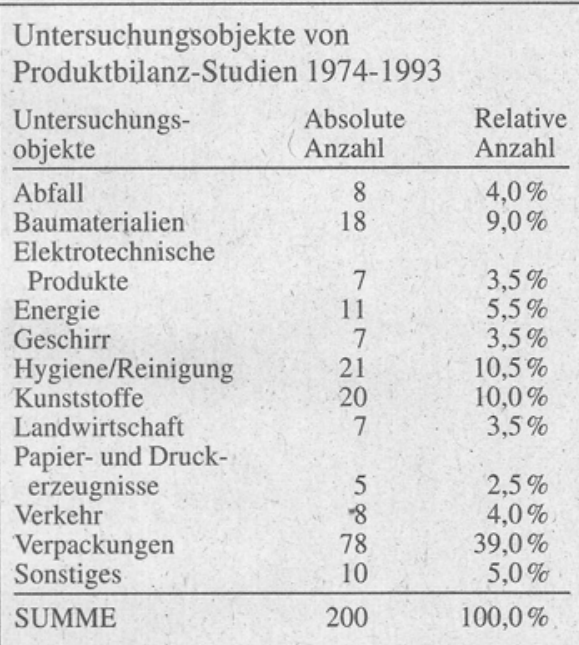


(c) 20I0 Authors; licensee IÖW and oekom verlag. This is an article distributed under the terms of the Creative Commons Attribution Non-Commercial No Derivates License (http://creativecommons.org/licenses/by-nc-nd/3.o/), which permits unrestricted use, distribution, and reproduction in any medium, provided the original work is properly cited. 